\title{
Modeling Motion, Ablation and Energy Deposition of Meteoroid in the Atmosphere Taking Account of the Curved Trajectory
}

\author{
I.G. Brykina, L.A. Egorova \\ Institute of Mechanics of Lomonosov Moscow State University, \\ Moscow, 119192, Russia \\ shantii@mail.ru
}

\begin{abstract}
The problem of modeling the motion, ablation, and energy release of a meteoroid or its fragments moving as a single body is considered. A computer program for calculating the system of meteor physics equations by the Runge-Kutta method is created and tested. The equations take into account the curvilinearity of the trajectory of meteor body, gravity, and change of the heat transfer coefficient along the trajectory. Test calculations were performed for meteor bodies of various sizes moving in the atmosphere. Change of the trajectory angle with respect to the horizon depending on the entry parameters is shown. The effect of taking into account the variability of the trajectory angle on the change of the velocity, mass loss, kinetic energy and trajectory of the meteoroid is estimated.
\end{abstract}

Keywords: meteoroid, ablation, energy release, curved trajectory.
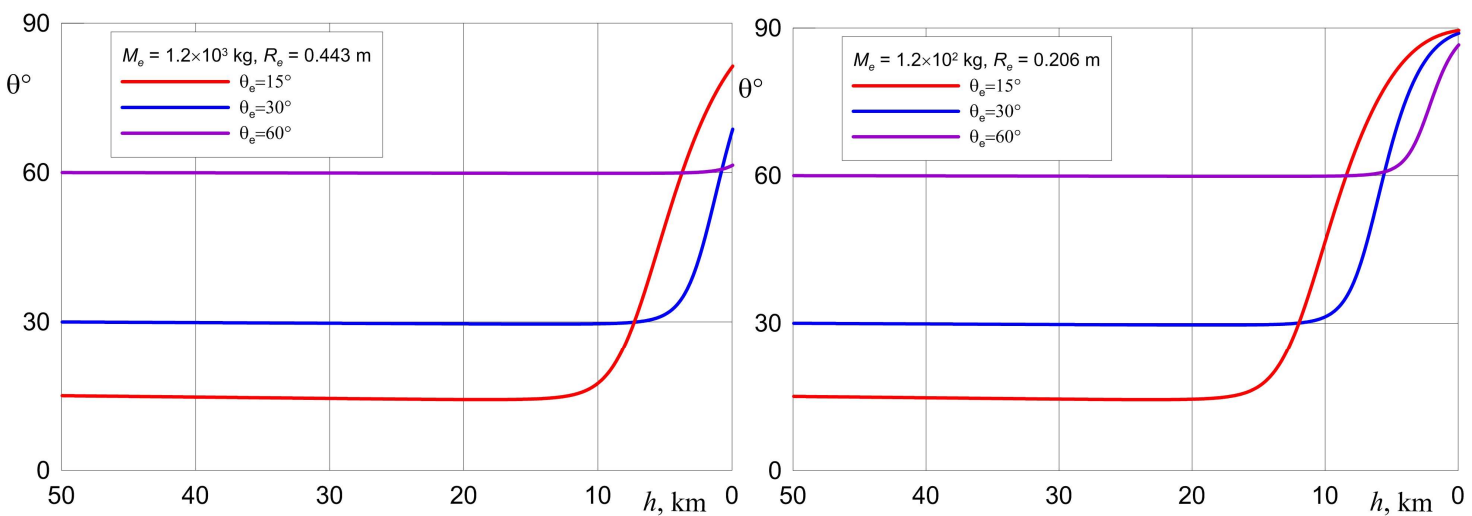

Changing the trajectory angle with respect to the horizon for different entry masses and angles 


\title{
Моделирование движения, абляции и энерговыделения метеороида в атмосфере с учетом криволинейности траектории
}

\author{
И.Г. Брыкина, Л.А. Егорова \\ НИИ механики МГУ им. М.В. Ломоносова, Москва, 119192, Мичуринский пр., 1 \\ egorova@imec.msu.ru
}

\begin{abstract}
Аннотация
Рассматривается задача моделирования движения, абляции и энерговыделения метеороида или его фрагментов, движущихся как единое тело. Создана и протестирована программа численного расчета системы уравнений метеорной физики методом РунгеКутты. В уравнениях учитывается криволинейность траектории метеорного тела, сила тяжести и изменение коэффициента теплопередачи вдоль траектории. Проведены тестовые расчеты для движущихся в атмосфере метеорных тел разных размеров, показано изменение угла наклона траектории по отношению к горизонту в зависимости от начальных параметров при входе в атмосферу. Оценивается влияние учета переменности угла наклона траектории на изменение скорости, массы, кинетической энергии траекторию метеороида.

Ключевые слова: метеороид, абляция, энерговыделение, криволинейная траектория.
\end{abstract}

\section{1. Введение}

Прохождение естественных космических тел через атмосферу Земли с большими скоростями сопровождается процессами, не проявляющимися при обтекании космических аппаратов. Под действием значительных аэродинамических нагрузок, возрастающих по мере проникновения в более плотные слои атмосферы, большинство космических тел разрушается. Под действием сильных тепловых потоков, в основном радиационных для крупных тел, осколки астероидов или комет плавятся, испаряются и теряют большую часть своей массы. Для моделирования входа метеороида в атмосферу, воспроизведения его траектории и наблюдаемой световой кривой и оценки его неизвестной начальной массы, необходимо разработать подходящий сценарий его разрушения и модель его абляции.

Космические тела имеют разный состав, скорость, размер, плотность и структуру (включая наличие дефектов), и разрушаются они по-разному. Поэтому разрабатываются различные подходы к моделированию их разрушения. В одном подходе, предполагающем, что метеороид дробится на большое количество осколков, первая стадия их движения, когда они объединены общей ударной волной, описывается с использованием моделей облака фрагментов, движущихся как единое тело, которое деформируется под действием аэродинамических нагрузок [1-7 и др.]. Другой подход, основанный на предположении, что фрагменты раздробленного метеороида движутся независимо, каждый со своей ударной волной, включает в себя модели одностадийного и прогрессивного дробления [8-12 и др.]. В последние годы разрабатываются различные гибридные модели, комбинирующие разные типы фрагментации, включая разрушение на большие независимо движущиеся фрагменты и кластеры мелких фрагментов, движущихся совместно. Такие подходы использовались для моделирования разрушения, полета и световых кривых болидов, зарегистрированных наземными и спутниковыми системами наблюдений [13-17 и др.]. 
Процессы движения и абляции единого и дробящегося метеороида обычно моделируются с использованием системы уравнений метеорной физики, включающей в себя уравнения для скорости и уноса массы тела вдоль траектории, и для самой траектории. Эти уравнения применяются к метеороиду до начала его разрушения, к облаку фрагментов, движущемуся как единое тело, в случае применения первого подхода к моделированию разрушения, и к отдельным фрагментам раздробленного метеорода в случае их независимого движения. В последнем случае полный унос массы и энерговыделение метеороида определяется суммированием по всем фрагментам.

При моделировании взаимодействия метеорных тел с атмосферой в литературе часто используются уравнения простой физической теории метеоров $[18,19]$, в которых не учитываются сила тяжести и изменение угла наклона траектории по отношению к горизонту, т.е. траектория считается прямолинейной. Для моделирования абляции крупного метеороида необходимо знать радиационный тепловой поток к поверхности тела. Это очень сложная проблема, поскольку подавляющее большинство исследований по радиационному нагреву относятся к теплообмену возвращаемых космических аппаратов, и область рассматриваемых в них скоростей и размеров тел довольно ограничена. Поэтому в метеорной тематике коэффициент теплопередачи, точнее, зависящий от него коэффициент абляции, обычно полагается постоянным (иногда ступенчатой функцией). Часто он подбирается так, чтобы результаты расчетов соответствовали данным наблюдений за полетом и свечением космических тел. При постоянном коэффициенте абляции уравнения простой физической теории метеоров имеют аналитическое решение, определяющее в неявном виде массу и скорость метеороида в зависимости от начальных параметров $[18,19]$. В большинстве работ используются эти аналитические решения, и не возникает необходимости применять численные методы для решения дифференциальных уравнений.

В данной работе с целью моделирования скорости, уноса массы и энерговыделения метеороида создана программа решения расширенных уравнений метеорной физики методом Рунге - Кутты. Эти уравнения отличаются от уравнений простой физической теории метеоров, которые решались в предыдущих работах авторов [6, 7 и др.] тем, что в них учитывается сила тяжести и криволинейность траектории, т.е. добавляется дополнительный член в уравнение движения и дополнительное дифференциальное уравнение для угла наклона траектории. Для коэффициента теплопередачи в уравнении абляции используется аппроксимационная зависимость от скорости тела, его размера и плотности атмосферы, т.е. учитывается его изменение вдоль траектории. Исследуется целесообразность учета силы тяжести и криволинейности траектории путем оценки их влияния на характеристики взаимодействия метеороида с атмосферой.

\section{2. Постановка задачи}

\section{1. Система основных уранений}

Будем рассматривать торможение, абляцию и энерговыделение в атмосфере метеороида, движущегося как единое тело, или отдельного фрагмента в случае его разрушения. Уравнение движения, задающее изменение скорости центра масс метеорного тела с учетом силы аэродинамического сопротивления и силы тяжести, имеет вид [20]

$$
M \frac{\mathrm{d} V}{\mathrm{~d} t}=-\frac{1}{2} S C_{D} \rho V^{2}+M g \sin \theta
$$

Здесь $t$ - время; $V, M$ и $S$ - скорость, масса и площадь миделева сечения метеороида; $\rho-$ плотность воздуха; $C_{D}$ - коэффициент лобового сопротивления; $\theta$ - угол наклона траектории по отношению к горизонту (к касательной к поверхности Земли); $g$ - ускорение свободного падения. При метеорных скоростях для небольших метеорных тел сила сопротивления воздуха значительно превышает силу тяжести, поэтому ее не учитывают в простой физической 
теории метеоров. Однако для крупных метеорных тел, осколки которых не испаряются полностью и долетают до земной поверхности, учет силы тяжести существенен в нижних слоях атмосферы, где скорости этих осколков (или всего метеорного тела) вследствие торможения существенно уменьшаются.

Уравнение абляции (уноса массы) метеорного тела имеет вид

$$
Q \frac{\mathrm{d} M}{\mathrm{~d} t}=-\frac{1}{2} S C_{H} \rho V^{3}
$$

Здесь $Q$ - эффективная теплота уноса массы; $C_{H}$ - коэффициент теплопередачи, приходящийся на единицу площади миделева сечения.

Движение метеороида вдоль траектории с переменным углом наклона $\theta$ описывается следующими уравнениями [21]:

$$
\begin{gathered}
\frac{\mathrm{d} h}{\mathrm{~d} t}=-V \sin \theta, \\
\frac{\mathrm{d} \theta}{\mathrm{d} t}=\frac{g \cos \theta}{V}-\frac{V \cos \theta}{R_{\otimes}+h}
\end{gathered}
$$

Здесь $h$ - высота полета; $R \otimes-$ радиус Земли. Под действием земного притяжения первоначальное направление движения метеорного тела искривляется по направлению к центру Земли. Второй член в правой части уравнения (4) с противоположным знаком связан с учетом кривизны поверхности Земли.

Будем использовать модель изотермической атмосферы

$$
\rho=\rho_{0} \exp \left(-\frac{h}{h^{*}}\right)
$$

где $\rho_{0}=1.29$ кг/м² - плотность атмосферы на уровне моря; $h^{*}=7$ км - шкала высот.

Для определения $V, M, \theta, h$ в зависимости от $t$ надо решить пять уравнений (1)-(5) с начальными условиями при $t=0: h=h_{e}, \rho=\rho_{e}, V=V_{e}, M=M_{e}, \theta=\theta_{e}$.

Будем полагать, что унос массы метеороида происходит так, что форма тела не меняется. Тогда связь площади миделя тела $S$ с его массой $M$ и плотностью $\delta$ можно выразить через коэффициент формы $f$ [22]

$$
f=\frac{S}{(M / \delta)^{2 / 3}}
$$

Перейдем к новой независимой переменной $\rho$ с использованием соотношения

$$
\frac{\mathrm{d}}{\mathrm{d} t}=\frac{\rho V \sin \theta}{h^{*}} \frac{\mathrm{d}}{\mathrm{d} \rho}
$$

Уравнения для $V, M, \theta$ с учетом соотношений (6) и (7) примут вид

$$
\begin{gathered}
\frac{\mathrm{d} V}{\mathrm{~d} \rho}=-\frac{1}{2} \frac{f h^{*} C_{D}}{\delta^{2 / 3} \sin \theta} V M^{-1 / 3}+\frac{g h^{*}}{\rho V}, \frac{\mathrm{d} M}{\mathrm{~d} \rho}=-\frac{1}{2} \frac{f h^{*} C_{H}}{\delta^{2 / 3} \sin \theta Q} V^{2} M^{2 / 3}, \\
\frac{\mathrm{d} \theta}{\mathrm{d} \rho}=\frac{h^{*}}{\rho V^{2} \operatorname{tg} \theta}\left(g-\frac{V^{2}}{R_{\otimes}+h}\right)
\end{gathered}
$$

Введем безразмерные переменные 


$$
v=\frac{V}{V_{e}}, \quad m=\frac{M}{M_{e}}, \quad \bar{\rho}=\frac{\rho}{\rho_{0}}
$$

и безразмерные коэффициенты $A_{1}, A_{2}, A_{3}$

$$
A_{1}=\frac{1}{2} \frac{f h^{*} C_{D} \rho_{0}}{\delta^{2 / 3} M_{e}^{1 / 3}}, \quad A_{2}=A_{1} \sigma V_{e}^{2}, \quad A_{3}=\frac{g h^{*}}{V_{e}^{2}}, \quad \sigma=\frac{C_{H}}{Q C_{D}},
$$

где $\sigma$ - коэффициент абляции, который обычно используется в метеорной физике и чаще всего полагается постоянным, представим уравнения (8) в безразмерном виде, удобном для численного интегрирования

$$
\begin{gathered}
\frac{\mathrm{d} v}{\mathrm{~d} \bar{\rho}}=-A_{1} \frac{v m^{-1 / 3}}{\sin \theta}+A_{3}(\bar{\rho} v)^{-1}, \quad \frac{\mathrm{d} m}{\mathrm{~d} \bar{\rho}}=-A_{2} \frac{v^{2} m^{2 / 3}}{\sin \theta}, \\
\frac{\mathrm{d} \theta}{\mathrm{d} \bar{\rho}}=\frac{1}{\bar{\rho} \operatorname{tg} \theta}\left(\frac{A_{3}}{v^{2}}-\frac{h^{*}}{R_{\otimes}+h}\right)
\end{gathered}
$$

Для решения этих уравнений надо задать начальные условия, форму метеороида или его фрагмента, коэффициент лобового сопротивления, эффективную теплоту уноса массы и коэффициент теплопередачи. В данной работе полагалось, что метеорное тело имеет сферическую форму, при этом коэффициент формы $f=1.21$. Коэффициент лобового сопротивления $C_{D}$ для шара полагался постоянным, равным 1 или 0.92. Эффективная теплота уноса массы $Q$ также полагалась постоянной, равной $6 \mathrm{~km}^{2} / \mathrm{c}^{2}$ или $8 \mathrm{~km}^{2} / \mathrm{c}^{2}$ (теплоте испарения обыкновенных хондритов [8]).

Коэффициент радиационной теплопередачи на единицу площади миделя $C_{H}$ вдоль траектории метеорного тела рассчитывался по аппроксимационной формуле

$$
C_{H}=\psi \varphi C_{H 0}(V, R, \rho) .
$$

Здесь параметр $\varphi$, характеризующий изменение теплового потока вдоль поверхности сферы, полагался равным $0.7 ; C_{H 0}-$ коэффициент теплопередачи в точке торможения сферы для неразрушающейся поверхности, определялся по формуле [23] в зависимости от скорости тела $V$, радиуса $R$ и плотности атмосферы $\rho$ с дополнительной поправкой по скорости $V$ для лучшего согласования с известными численными решениями при небольших значениях $V$. Отметим, что существует неопределенность в значении радиационного потока к телу, связанная с неучтенным влиянием на него опережающего излучения, турбулентности, с экранированием его парами метеороида, с неопределенностью в оптических свойствах горячего воздуха и паров, неизвестной формой тела, а также с влиянием других факторов. В исследовании [24] отмечалось, что такая неопределенность в точке торможения составляет примерно от +81 до - 52 \% для входа в атмосферу Земли со скоростью 15 км/с. Поэтому в выражение для коэффициента теплопередачи введен фактор неопределенности $\psi$. В данной работе расчеты проводились в основном при $\psi=1$, поскольку такое значение этого параметра давало наилучшее согласование расчетной и наблюдательной кривой энерговыделения Челябинского болида [25].

\section{2. Энерговыделение метеоного тела в атмосфере}

Один из наблюдаемых параметров при движении метеороида в атмосфере - интенсивность свечения $I$, которая определяется в зависимости от изменения его кинетической энергии $J[10]$

$$
I=\tau J, \quad J=-\frac{\mathrm{d} E}{\mathrm{~d} t}=-\frac{\mathrm{d}}{\mathrm{d} t}\left(\frac{M V^{2}}{2}\right)=-\left(\frac{V^{2}}{2} \frac{\mathrm{d} M}{\mathrm{~d} t}+M V \frac{\mathrm{d} V}{\mathrm{~d} t}\right)
$$


Здесь $\tau$ - коэффициент эффективности излучения. Выделяемую метеороидом кинетическую энергию $E$ в единицу времени за счет потери скорости и массы можно рассчитать как

$$
\begin{aligned}
J & =-\frac{\rho V \sin \theta}{h^{*}}\left(\frac{V^{2}}{2} \frac{\mathrm{d} M}{\mathrm{~d} \rho}+M V \frac{\mathrm{d} V}{\mathrm{~d} \rho}\right)=\frac{\rho V^{3}}{2} \frac{f C_{D}}{\delta^{2 / 3}} M^{2 / 3}\left(\frac{V^{2}}{2} \sigma+1\right)-g \sin \theta M V= \\
& =\frac{\rho_{0} V_{e}^{3} M_{e}^{2 / 3} f C_{D}}{2 \delta^{2 / 3}} \bar{\rho} v^{3} m^{2 / 3}\left(\frac{\sigma V_{e}^{2}}{2} v^{2}+1\right)-g M_{e} V_{e} \sin \theta m v
\end{aligned}
$$

В литературе часто используется понятие выделения кинетической энергии на единицу высоты, которое находится по формуле

$$
\frac{\mathrm{d} E}{\mathrm{~d} h}=\frac{J}{V \sin \theta}=\frac{\rho_{0} V_{e}^{2} M_{e}^{2 / 3} f C_{D}}{2 \delta^{2 / 3} \sin \theta} \bar{\rho} v^{2} m^{2 / 3}\left(\frac{\sigma V_{e}^{2}}{2} v^{2}+1\right)-g M_{e} m
$$

\section{3. Метод расчета и его тестирование}

Для системы уравнений (5), (12) была составлена программа расчета методом РунгеКутты 4-го порядка точности [26]. Начальные данные задавались на некоторой высоте $h_{e}$. Использовался равномерный шаг $\Delta h$ по высоте (высота менялась от $h_{e}$ до 0 (поверхность Земли) или до некоторой конечной высоты $h_{k}$ ). Соответственно шаг по переменной безразмерной плотности $\bar{\rho}$ был неравномерным:

$$
\Delta \rho=\exp \left(\frac{h_{z}-h}{h^{*}}\right)-\exp \left(-\frac{h}{h^{*}}\right)
$$

Для оценки точности расчета скорости и массы метеороида вдоль траектории проводились тестовые расчеты с разным шагом по высоте.

Коэффициент теплопередачи $C_{H}$ на каждом шаге рассчитывался с помощью специально созданной подпрограммы, при этом значения радиуса и скорости брались с предыдущего шага. На каждом шаге, после определения $m, v$ и $\theta$, рассчитывалось энерговыделение метеороида по формулам (13) и (14).

Для верификации созданной программы проводились тестовые расчеты и их сравнение с имеющимися численными и аналитическими решениями уравнений простой физической теории метеоров. Поэтому тестовые расчеты проводились для тех же условий: предполагалась прямолинейная траектория движения метеороида, т.е. не учитывалось изменение угла наклона траектории к горизонту, и пренебрегалось влиянием силы тяжести.

Первый тестовый расчет проводился при постоянном коэффициенте теплопередачи и постоянном коэффициенте абляции $\sigma$. В этом случае имеется точное аналитическое решение уравнений ФТМ $[18,19]$. На рис. 1 проводится сравнение изменения скорости и массы метеороида вдоль траектории в зависимости от высоты полета с аналитическим решением и численным решением, полученным методом Рунге - Кутты третьего порядка М.Д. Брагиным. Начальные данные соответствовали условиям входа в атмосферу Челябинского метеороида [15]: $\delta=3.3 \times 10^{3} \mathrm{\kappa г} / \mathrm{m}^{3}, V_{e}=19 \mathrm{\kappa м} / \mathrm{c}, \theta=18^{\circ}$; полагалось $M_{e}=1.5 \times 10^{7} \mathrm{\kappa г,}, C_{D}=1, C_{H}=0.1$, $Q=8 \mathrm{\kappa м}^{2} / \mathrm{c}^{2} ; h_{e}=80 \mathrm{\kappa м}, h_{k}=5$ км, $\Delta h_{z}=0.5$ км. Расчет соответствует моделированию движения и абляции метеороида, движущегося как единое тело, не разрушаясь.

Во втором тестовом расчете полагалось: $M_{e}=300$ кг, $Q=6 \mathrm{kм}^{2} / \mathrm{c}^{2} ; h_{e}=50$ км, $h_{k}=25$ км; остальные параметры не менялись. Этот расчет соответствует моделированию движения и абляции гипотетического фрагмента (весом 300 кг) Челябинского метеороида при его разрушении на высоте 50 км. На рис. 2 проводится сравнение с точным аналитическим решением, полученным М.Д. Брагиным. 

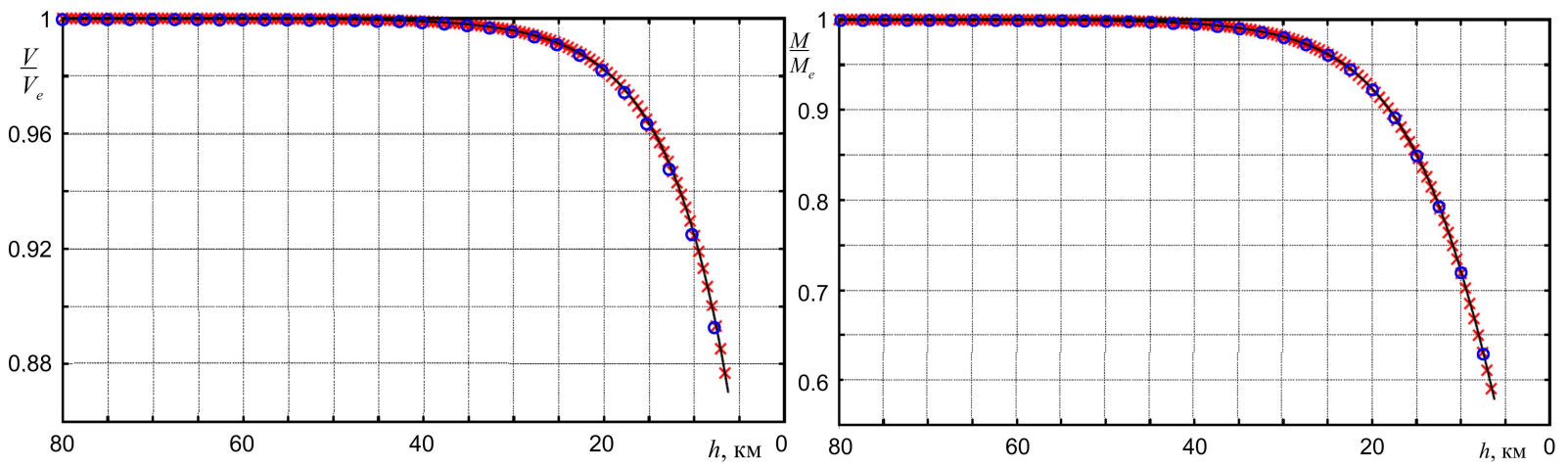

Рис. 1. Сравнение результатов расчета массы и скорости (синие кружки) с точным аналитическим решением и численными расчетами М.Д. Брагина (черные линии и красные кресты) при одних и тех же начальных данных, определяющих параметрах и шаге по высоте
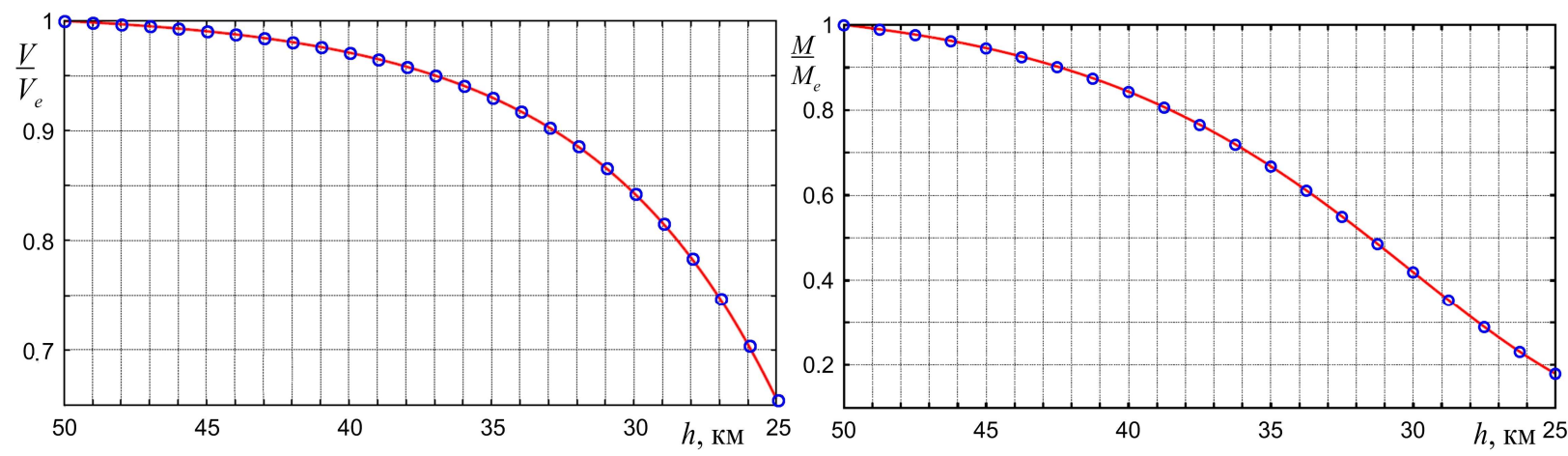

Рис. 2. Сравнение результатов расчета массы и скорости (синие кружки) с точным аналитическим решением, полученным М.Д. Брагиным (красные линии) при одних и тех же начальных данных, определяющих параметрах и шаге по высоте

Рисунки 1 и 2 показывают, что скорость и масса метеороида вдоль траектории, рассчитанные по созданной программе, хорошо совпадают с известными решениями.

В третьем тестовом варианте расчетов проводится сравнение численного решения для энерговыделения метеороида в атмосфере и его уноса массы с аналитическим решением, полученным в работе [27] в предположении, что масса метеороида меняется гораздо быстрее его скорости. Тот факт, что до начала существенного торможения масса тела убывает быстрее, чем его скорость, неоднократно отмечался в литературе, это видно также из расчетов, представленных на рис. 3 .
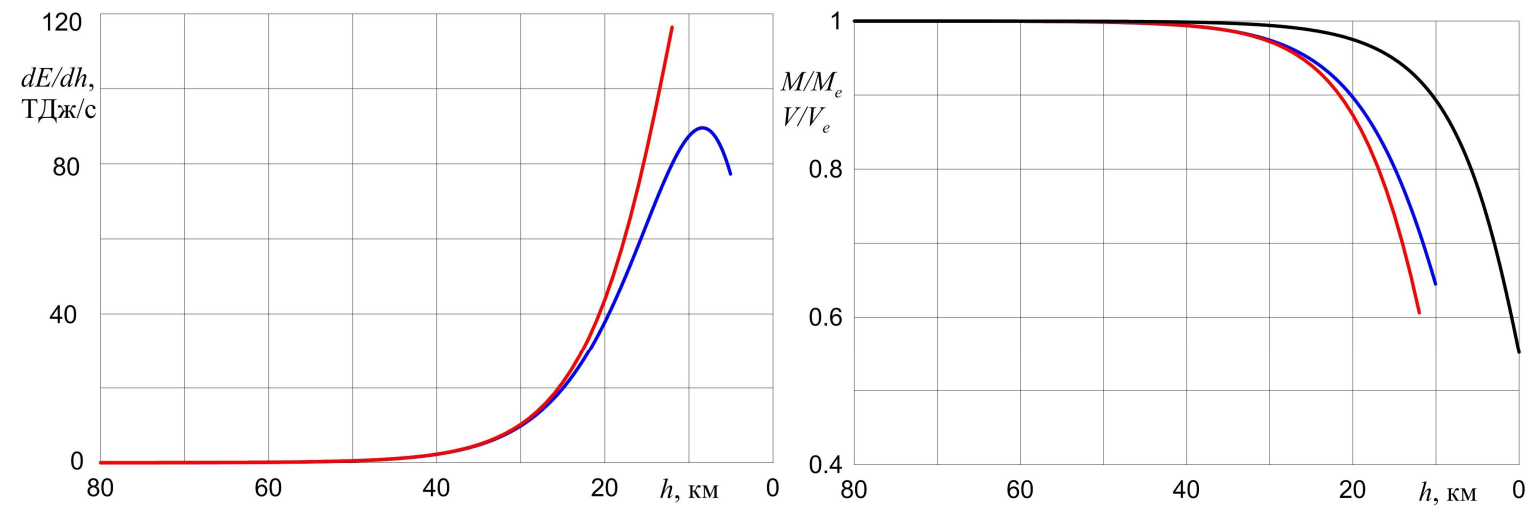

Рис. 3. Сравнение результатов численного расчета (синие кривые) массы и энерговыделения метеороида с аналитическим решением[27] (красные). Черная кривая - результат расчета скорости 
На этом рисунке приведены энерговыделение метеороида на единицу высоты, его масса и скорость, рассчитанные по созданной программе, а также расчеты по формулам [27]. Расчеты проведены при следующих значениях параметров: $\delta=3.2 \times 10^{3}$ кг $/ \mathrm{m}^{3}, V_{e}=18.8 \mathrm{\kappa м} / \mathrm{c}$, $\theta=15^{\circ}, M_{e}=0.977 \times 10^{7}$ кг, $C_{D}=1, Q=8 \mathrm{\kappa m}^{2} / \mathrm{c}^{2} ; h_{e}=80$ км. Коэффициент теплопередачи соответствовал работе [26], в которой $C_{H 0}$ рассчитывался по формуле для радиационного теплового потока в точке торможения работы[28], коэффициент $\psi=0.7$.

Видно, что на высотах более 20 км изменение скорости метеороида незначительно и численное и аналитическое решения для уноса массы и энерговыделения очень близки. На высотах менее 20 км решения начинают расходиться и аналитическое решение перестает быть справедливым, т.к. оно не учитывает изменение скорости и ее производную.

\section{4. Влияние силы тяжести и криволинейности траектории метеороида на его скорость, унос массы и энерговыделение}

Для очень крупных метеорных тел сила тяжести сравнима с силой аэродинамического сопротивления не только в нижних слоях атмосферы, где скорости становятся небольшими, но и на больших высотах, вследствие того, что плотность атмосферы мала, а масса тела до начала его разрушения велика (см. уравнение 1). Так, расчеты показывают, что для Челябинского метеороида с массой 12000 т высотах более $45 \div 50$ км (высота начала его разрушения) сила тяжести превосходит силу аэродинамического сопротивления, что приводит даже к небольшому увеличению скорости метеороида, которое видно на рис. 4. Такое временное превышение входной скорости было зарегистрировано системами наблюдений для Челябинского метеороида [15]. Однако расчеты показывают, что это незначительное увеличение скорости вследствие учета силы тяжести на больших высотах практически не влияет на дальнейший расчет скорости, массы и энерговыделения метеороида. Учет силы тяжести важен в нижних слоях атмосферы, где скорости небольших метеорных тел (осколков крупных тел) вследствие значительного торможения уменьшаются и приближаются к нулю. Затем, на последнем участке своей траектории эти фрагменты под действием силы тяжести падают на землю в виде метеоритов.

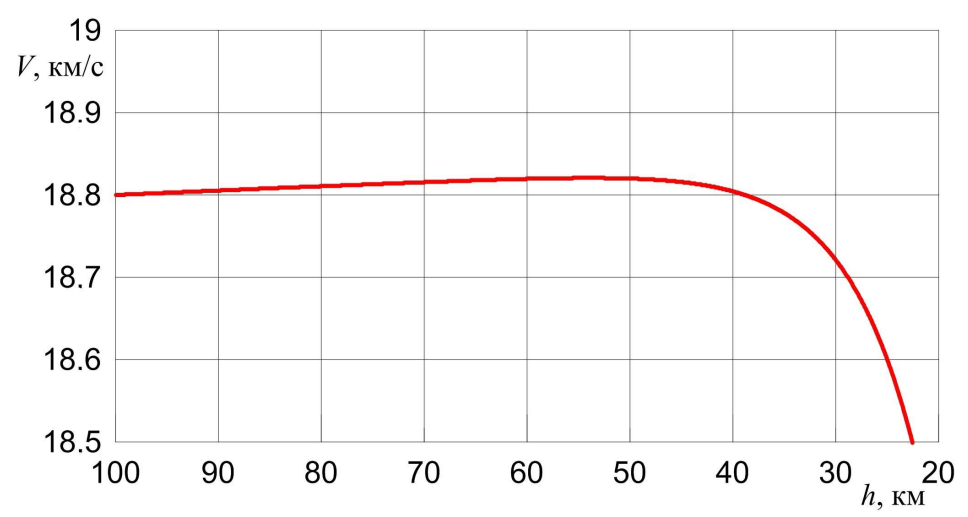

Рис. 4. Изменение вдоль траектории скорости метеороида, входящего в атмосферу с начальными параметрами, соответствующими рис. 3

Скорость изменения угла наклона траектории $\theta$ описывается уравнением (4). Зависимость производной $\theta$ по времени от скорости полета представлена на рис. 5. Нетрудно получить из уравнения (4), что производная $\theta$ обращается в 0 при скорости, примерно равной 7.9 км/с. Таким образом, для любых метеороидов, входящих в атмосферу с разными скоростями и массами и под разными углами, угол наклона их траектории по отношению к горизонту сначала медленно уменьшается до тех пор, пока их скорость не достигнет 7.9 км/с. Затем с уменьшением скорости он начинает быстро увеличиваться, и с приближением скорости к 
нулю стремится к 90 (вертикальное движение).Приведенные ниже расчеты входа в атмосферу разных метеорных тел подтверждают эти выводы, следующие из анализа кривой на рис. 5.

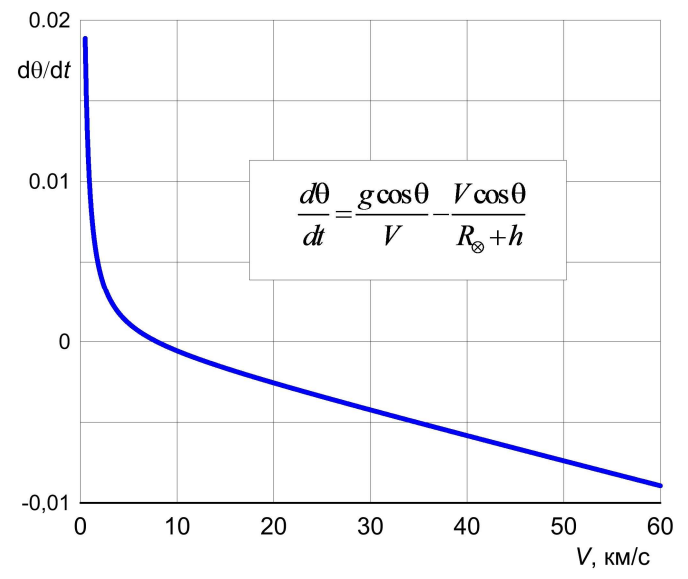

Рис. 5. Зависимость производной $\theta$ по времени от скорости полета при $\theta=15^{\circ}$ и $h \ll R_{\otimes}$

Тестовые расчеты проведены для тел разной начальной массы, входящих в атмосферу под разными углами. Начальная скорость и плотность тела соответствовали условиям входа в атмосферу Челябинского метеороида [15]: $\delta=3.3 \times 10^{3}$ кг $/ \mathrm{m}^{3}, V_{e}=19$ км/c; $Q$ полагалось равным $6 \mathrm{kм}^{2} / \mathrm{c}^{2}$; коэффициент теплопередачи $C_{H}$ рассчитывался по формуле (12). Результаты расчетов представлены на рис. 6 для трех углов входа метеорного тела в атмосферу: $\theta_{e}=15^{\circ}, 30^{\circ}, 60^{\circ}$ и четырех начальных значениях массы $M_{e}=1.2 \times 10^{7}$ кг $(a), 1.2 \times 10^{5}$ кг (б), $1.2 \times 10^{3}$ кг (в), $1.2 \times 10^{2}$ кг (2).
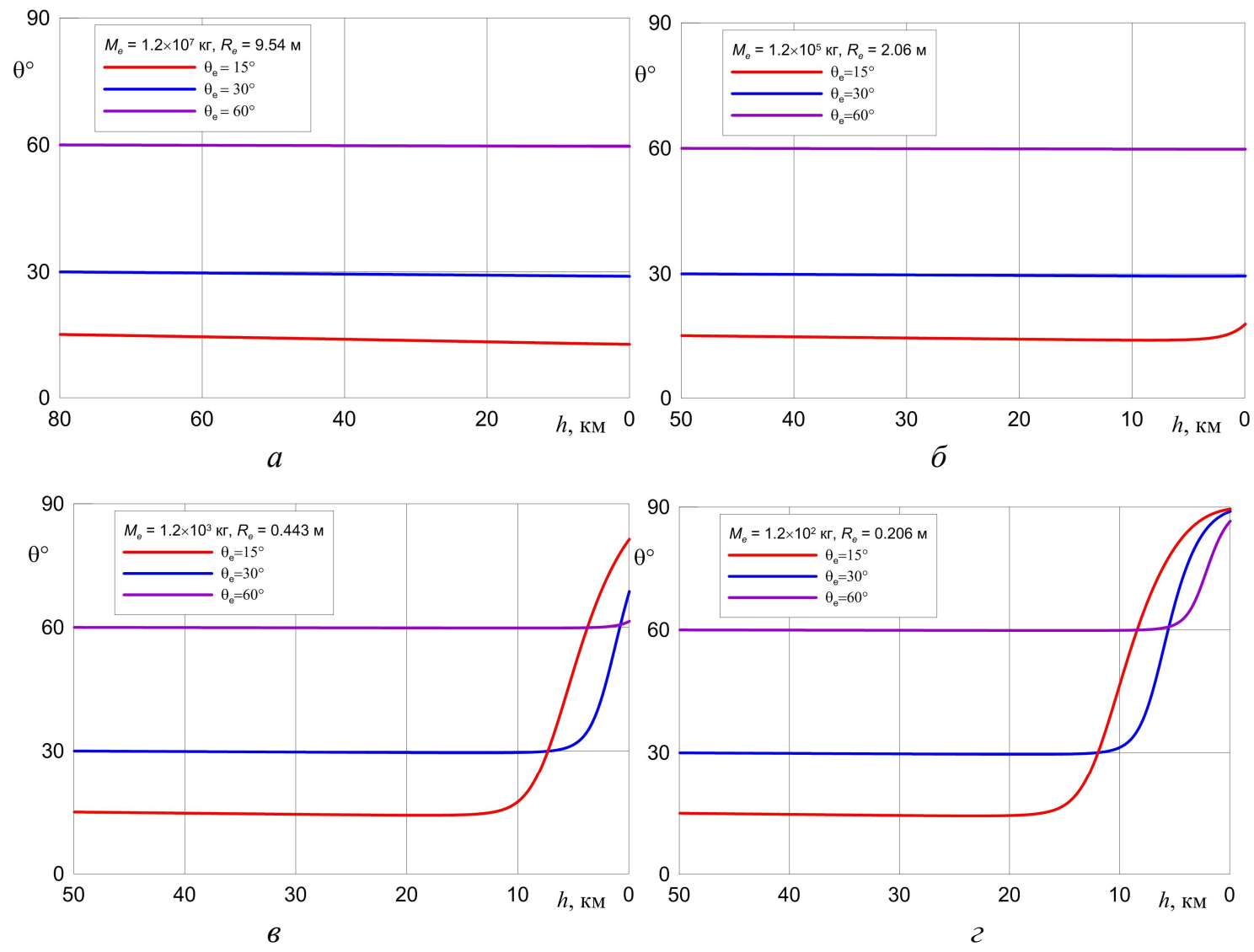

Рис. 6. Изменение угла наклона траектории при разных начальных массах и углах 
Расчет для самой большой массы соответствует гипотетическому движению Челябинского метеороида без его разрушения, $h_{e}=80$ км; расчеты для меньших масс - движению гипотетических осколков (возможно, реальных) Челябинского метеороида после его разрушения, которое началось на высоте 50 км.

Рисунок 6 демонстрирует, что в случае крупных тел с радиусом примерно более $1 \mathrm{~m}$ траектория полета является практически прямолинейной и в расчетах можно полагать угол наклона траектории постоянным и равным углу входа в атмосферу. Однако следует учитывать, что такие тела обычно дробятся в атмосфере на более мелкие фрагменты, поэтому их траекторию можно считать прямолинейной только до начала разрушения, когда рассматривается их движение как единого тела. Для тел или фрагментов с радиусом менее 1 м и небольших углов входа в атмосферу траектория заметно искривляется на низких высотах. Чем меньше масса тела и меньше начальный угол $\theta_{e}$, тем на большей высоте начинается существенный рост угла $\theta$, т.е. раньше начинается искривление траектории.
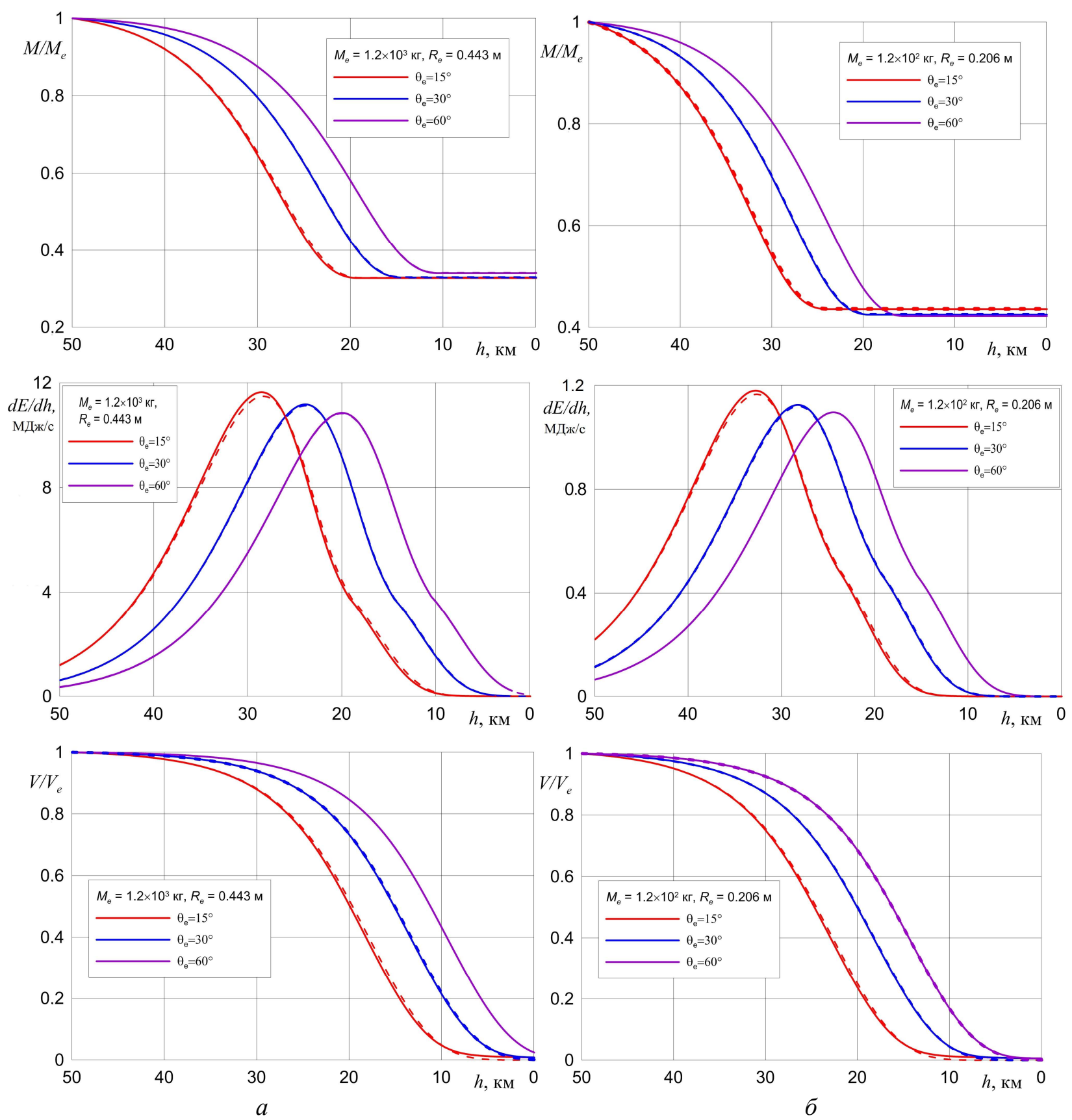

Рис. 7. Изменение массы, энерговыделения и скорости метеорного тела вдоль криволинейной (сплошные линии) и прямолинейной (штриховые) траекторий при $M_{e}=1.2 \times 10^{3}$ кг (a) и $1.2 \times 10^{2}$ кг (б) 
На рис. 7 приведены результаты расчета скорости, массы и энерговыделения метеорного тела на единицу высоты при начальных значениях массы $M_{e}=1.2 \times 10^{3}$ кг и $1.2 \times 10^{2}$ кг и при разных углах входа для криволинейной и прямолинейной траекторий. Видно, что увеличение угла входа тела в атмосферу $\theta_{e}$ приводит к более медленному его торможению, к более позднему прекращению абляции (уноса массы тела), сдвигу высоты максимума энерговыделения на более низкие высоты и некоторому уменьшению самого максимума. Рисунок 7 показывает также, что учет криволинейности траектории приводит к более медленному торможению тела и, соответственно, к большей его скорости на низких высотах, на последнем участке траектории. В то же время, учет изменения угла $\theta$ вдоль траектории оказывает слабое влияние на изменение массы и энерговыделение метеороида даже при малом угле $\theta_{e}=15^{\circ}$. Это связано с тем, что значительное увеличение угла $\theta$ начинается, когда скорость метеороида снижается примерно до 2 км/с, что можно видеть и из рис. 5 , а при такой небольшой скорости абляция уже прекращается и энерговыделение становится незначительным.

Существенное влияние учет изменения угла $\theta$ вдоль траектории оказывает на саму траекторию. На рис. 8 приведены рассчитанные траектории метеорных тел с начальными массами $M_{e}=1200,120$ и 12 кг при угле входа в атмосферу $15^{\circ}$, и для сравнения - прямолинейная траектория с постоянным углом $\theta=15^{\circ}$.

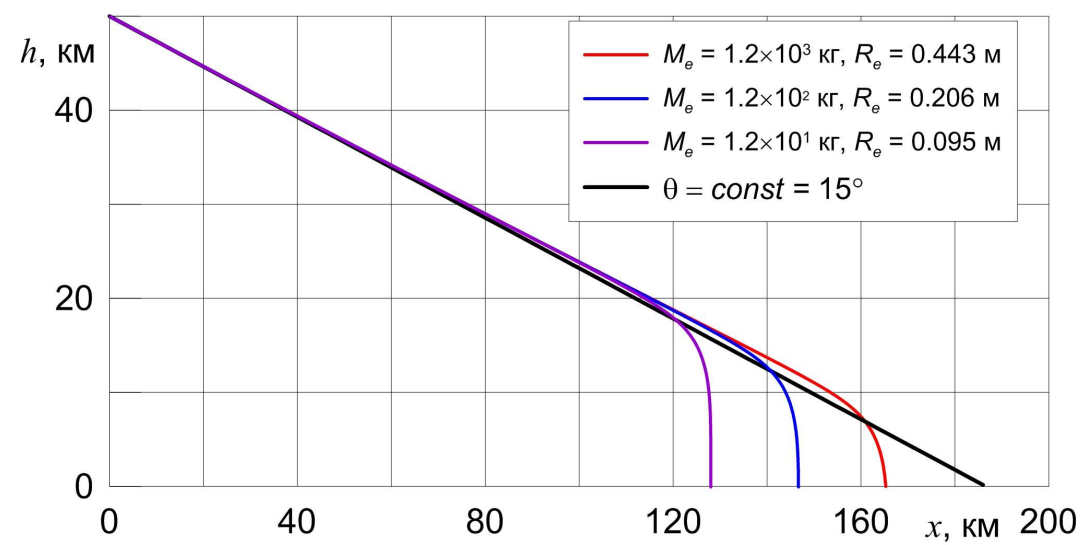

Рис. 8. Влияние учета изменения угла $\theta$ на траектории метеорных тел с разными начальными массами и начальным углом $15^{\circ}$. Черная линия - прямолинейная траектория, $x$ - горизонтальное расстояние вдоль поверхности Земли

При учете криволинейности траектории метеорное тело приближается к земной поверхности под углом, для небольших масс близким к $90^{\circ}$. Неиспарившееся метеорное тело выпадает на землю в виде метеорита, на рис. 8 - в точке пересечения его траектории с осью $x$ (расстояние вдоль горизонтальной поверхности Земли). Видно, что расстояние между точками падения метеорного тела на землю в случаях учета и неучета изменения угла $\theta$ вдоль траектории равняется примерно 21, 40 и 58.6 км при $M_{e}=1200,120$ и 12 кг соответственно. Таким образом, учет криволинейности траектории важен для определения примерных мест выпадения метеоритов, для построения их поля рассеяния.

\section{5. Заключение}

Создана и протестирована программа численного решения методом Рунге-Кутты системы уравнений метеорной физики, позволяющая рассчитывать скорость, унос массы, энерговыделение и траекторию метеорного тела с учетом ее криволинейности. Проведены тестовые расчеты для тел с разной начальной массой, входящих в атмосферу под разными углами. Показано, что в случае метеорных тел с радиусом менее 1 м при небольших углах входа относительно горизонта траектория движения заметно искривляется при существенном снижении скорости. Учет криволинейности траектории метеорного тела оказывает слабое влияние 
на изменение его массы и энерговыделения, таким образом при расчете этих характеристик взаимодействия тела с атмосферой траекторию можно считать прямолинейной. В то же время изменение угла наклона траектории оказывает существенное влияние на саму траекторию, поэтому его важно учитывать для оценки мест выпадения метеоритов при определении районов поиска выпавших фрагментов разрушенных космических тел.

\section{Благодарности и ссылки на гранты}

Работа выполнена в соответствии с планом исследований НИИ механики МГУ при частичной поддержке гранта РФФИ № 18-01-00740.

\section{Литература}

1. Григорян С.С. О движении и разрушении метеоритов в атмосферах планет // Космич. исслед. 1979. T. 17. № 6. C. $875-893$.

2. Melosh H.J. Atmospheric breakup of terrestrial impactors // Proc. Lunar Planet. Sci. 1981. V. 12A. Pp. 29-35.

3. Chyba C.F., Thomas P.J., Zahnle K.J. The 1908 Tunguska explosion - Atmospheric disruption of a stony asteroid // Nature. 1993. V. 361. Pp. 40-44.

4. Hills J.G., Goda M.P. The fragmentation of small asteroids in the atmosphere // Astron. J. 1993. V. 105. № 3. Pp. 1114-1144.

5. $\quad$ Григорян С.С., Ибодов Ф.С., Ибадов С.И. Челябинский суперболид: к физике взрыва // Астрон. вестн. 2013. Т. 47. № 4. С. 292-298.

6. Брыкина И.Г. О модели фрагментации крупного метеороида: моделирование взаимодействия Челябинского метеороида с атмосферой // Астрон. вестн. 2018. Т. 52. № 5. С. 437-446.

7. Брыкина И.Г., Брагин М.Д., Егорова Л.А. О моделях фрагментации метеороидов в атмосфере // Физико-химическая кинетика в газовой динамике. 2019. Т. 20. Вып. 2. http:/chemphys.edu.ru/issues/2019-20-2/articles/822/.

8. Baldwin B., Sheaffer Y. Ablation and breakup of large meteoroids during atmospheric entry // J. Geophys. Res. 1971. V. 76. № 19. Pp. 4653-4668.

9. Немчинов И.В., Попова О.П. Анализ Сихотэ-Алинского события 1947 г. и его сравнение с явлением 1 февраля 1994 г. // Астрон. вестн. 1997. Т. 31. № 5. С. 458-471.

10. Ceplecha Z., Borovička J., Elford W.G., ReVelle D.O., Hawkes R.L., Porubcan V.Í., Šimek M. // Meteor phenomena and bodies. Space Sci. Rev. 1998. V. 84. Pp. 327-471.

11. ReVelle D.O. NEO fireball diversity: Energetics-based entry modeling and analysis techniques //Proc. International Astronomical Union Symposium, 2007. V. 236. Cambridge Univ. Press. Pp. 95-106.

12. Avramenko M.I., Glazyrin I.V., Ionov G.V., Karpeev A.V. Simulation of the airwave caused by the Chelyabinsk superbolide // J. Geophys. Res. Atmospheres. 2014. V. 119. Pp. 7035-7050.

13. Ceplecha Z., ReVelle D.O. Fragmentation model of meteoroid motion, mass loss, and radiation in the atmosphere //Meteorit. \& Planet. Sci. 2005. V.40. № 1. Pp. 35-54.

14. Borovička J., Toth J., Igaz A., Spurny P., Kalenda P., Haloda J., Svoren J., Kornos L., Silber E., Brown P., Husarik M. The Košice meteorite fall: Atmospheric trajectory, fragmentation, and orbit // Meteoritics and Planetary Sci. 2013. V. 48. № 10. Pp. 1757-1779.

15. Borovička J., Spurny P., Brown P., Wiegert P., Kalenda P., Clark D., Shrbeny L. The trajectory, structure and origin of the Chelyabinsk asteroidal impactor // Nature. 2013. V. 503. Pp. 235-237.

16. Wheeler L.F., Register P.J., Mathias D.L.A fragment-cloud model for asteroid breakup and atmospheric energy deposition. Icarus.2017. V. 295. Pp. 149-169.

17. Borovička J., Popova O. Spurný P. The Maribo CM2 meteorite fall—Survival of weak material at high entry speed // Meteorit. \& Planet. Sci. 2019. V. 54. Pp. 1024-1041.

18. Бронштэн В.А. Физика метеорных явлений. М.: Наука, 1981. 416 с. 
19. Стулов В.П., Мирский В.Н., Вислый А.И. Аэродинамика болидов. М.: Наука, 1995.236 с.

20. Мещерский И.В. Работы по динамике тел переменной массы. М.: Изд-во технико-теоретической литературы, 1952. $280 \mathrm{c}$.

21. Астапович И.С. Метеорные явления в атмосфере Земли. М.: Физ-Мат. Лит., 1958. 640 с.

22. Левин Б.Ю. Физическая теория метеоров и метеорное вещество в солнечной системе. М.: Издво АН СССР, 1956. 293 с.

23. Брыкина И.Г., Егорова Л.А. Аппроксимационные формулы для радиационного теплового потока при больших скоростях // Изв. РАН. МЖГ. 2019. № 4. С. 123-134.

24. Johnston C.O., Mazaheri A., Gnoffo P., Kleb B., Sutton K., Prabhu D., Brandis A.M., Bose D. Radiative heating uncertainty for hyperbolic Earth entry, part 1: flight simulation modeling and uncertainty. J. Spacecraft \& Rockets. 2013. V. 50. No 1. Pp. 19-38.

25. Brykina I.G., Bragin M.D. On models of meteoroid disruption into the cloud of fragments // Planetary \& Space Sci. 2020. V. 187.No 104942.

26. Турчак Л.И. Основы численных методов. М.: Наука, 1987. 320с.

27. Брыкина И.Г., Тирский Г.А. Унос массы и световая кривая крупного метеороида. Аналитическое решение// Прикл. матем. и механ. 2017. Т. 81. № 5. С. 571-592.

28. Suttles J.T., Sullivan E.M., Margolis S.B. Curve fits of predicted inviscid stagnation-point radiative heating rates, cooling factors, and shock standoff distances for hyperbolic earth entry. NASA TN D7622. 1974. $45 \mathrm{p}$.

\section{References}

1. Grigoryan, S. S., "Meteorites motion and destruction in planet atmospheres," Cosmic Res., Vol. 17, 1979, pp. 724-740. http://adsabs.harvard.edu/abs/1980CosRe..17..724G

2. Melosh, H. J., "Atmospheric breakup of terrestrial impactors," Proc. Lunar Planet. Sci., Vol. 12A 1981, pp. 29-35. http://adsabs.harvard.edu/full/1981mrbf.conf...29M

3. Chyba, C. F., Thomas, P. J., Zahnle, K. J., "The 1908 Tunguska explosion - Atmospheric disruption of a stony asteroid," Nature, Vol. 361, 1993, pp. 40-44. https://www.nature.com/articles/361040a0

4. Hills, J. G., Goda, M. P., "The fragmentation of small asteroids in the atmosphere," Astron. J., Vol. 105. No. 3, 1993, pp. 1114-1144. http://adsabs.harvard.edu/full/1993AJ...105.1114H

5. Grigoryan, S. S., Ibodov, F. S., Ibadov, S. I., "Physical mechanism of Chelyabinsk superbolide explotion," Solar Syst. Res., Vol. 47, 2013.pp. 268-274. https://doi.org/10.1134/S0038094613040151

6. Brykina, I. G., "Large meteoroid fragmentation: modeling the interaction of the Chelyabinsk meteoroid with the atmosphere," Solar Syst. Res., Vol. 52, 2018, pp. 426-434.

https://doi.org/10.1134/S0038094618050027

7. Brykina, I. G., Bragin, M. D., Egorova, L. A., "O modeljah fragmentacii meteoroidov v atmosphere," Fiziko-himicheskaja kinetika v gazovoj dinamike (Phisical-Chemical Kinetics in Gas Dynamics), Vol. 20, No. 2, 2019. (in Russian) http://chemphys.edu.ru/issues/2019-20-2/articles/822/

8. Baldwin, B., Sheaffer, Y., "Ablation and breakup of large meteoroids during atmospheric entry," $J$. Geophys. Res., Vol. 76, 1971, pp. 4653-4668.

https://agupubs.onlinelibrary.wiley.com/doi/abs/10.1029/JA076i019p04653

9. Nemchinov, I. V., Popova, O. P., "An analysis of the 1947 Sikhote-Alin event and a comparison with the phenomenon of February 1, 1994," Sol. Syst. Res., Vol. 31, 1997, pp. 408-420.

https://ui.adsabs.harvard.edu/abs/1997SoSyR..31..408N/abstract

10. Ceplecha, Z., Borovička, J., Elford, W. G., ReVelle, D. O., Hawkes, R. L., Porubcan, V. Í., Šimek, M., "Meteor phenomena and bodies," Space Sci. Rev., Vol. 84, 1998, pp. 327-471.

https://link.springer.com/article/10.1023\%2FA\%3A1005069928850 
11. ReVelle, D. O., "NEO fireball diversity: Energetics-based entry modeling and analysis techniques," Proc. International Astronomical Union Symposium, Vol. 236, 2007, pp. 95-106. Cambridge Univ. Press, Cambridge, United Kingdom. https://doi.org/10.1017/S1743921307003122

12. Avramenko, M. I., Glazyrin, I. V., Ionov, G. V., Karpeev, A. V., "Simulation of the airwave caused by the Chelyabinsk superbolide," J. Geophys. Res. Atmospheres, Vol. 119, 2014, pp. 7035-7050. https://doi.org/10.1002/2013JD021028

13. Ceplecha, Z., ReVelle, D. O., "Fragmentation model of meteoroid motion, mass loss, and radiation in the atmosphere," Meteorit. \& Planet. Sci., Vol. 40, 2005, pp. 35-54. https://doi.org/10.1111/j.1945-5100.2005.tb00363.x

14. Borovička, J., Toth, J., Igaz, A., Spurny, P., Kalenda, P., Haloda, J., Svoren, J., Kornos, L., Silber, E., Brown, P., Husarik, M., "The Košice meteorite fall: Atmospheric trajectory, fragmentation, and orbit," Meteorit. \& Planet. Sci., Vol. 48, 2013, pp. 1757-1779. https://doi.org/10.1111/maps.12078

15. Borovička, J., Spurný, P., Brown, P., Wiegert, P., Kalenda, P., Clark, D., Shrbený, L., “The trajectory, structure and origin of the Chelyabinsk asteroidal impactor," Nature, Vol. 503, 2013, pp. 235-237. https://doi.org/10.1038/nature12671

16. Wheeler, L. F., Register, P. J., Mathias, D. L., "A fragment-cloud model for asteroid breakup and atmospheric energy deposition," Icarus, Vol. 295, 2017, pp. 149-169.

https://doi.org/10.1016/j.icarus.2017.02.011

17. Borovička, J., Popova, O., Spurný P., "The Maribo CM2 meteorite fall—Survival of weak material at high entry speed," Meteorit. \& Planet. Sci., Vol. 54, 2019, pp. 1024-1041.

https://doi.org/10.1111/maps.13259

18. Bronshten, V. A., Fizika meteornyh yavlenij, M.: Nauka, 1981. 416 p.

19. Stulov, V. P., Mirskij, V. N., Vislyj, A. I., Aerodinamika bolidov, M.: Nauka, 1995, 236 p.

20. Meshcherskiy, I. V., Raboty po dinamike tel peremennoy massy, M.: Izd-vo tekhniko-teoreticheskoy literatury, 1952, $280 \mathrm{p}$.

21. Astapovich, I. S., Meteornyye yavleniya v atmosphere Zemli, M.: Fiz-Mat, Lit., 1958. 640 p.

22. Levin, B. Yu., Fizicheskaya teoriya meteorov i meteornoye veshchestvo v solnechnoy sisteme, M.: Izdvo ANSSSR, 1956. 293p.

23. Brykina, I. G., Egorova, L.A., "Approximation formulas for the radiative heat flux at high velocities," Fluid Dyn., Vol. 54, 2019, pp. 562-574. https://doi.org/10.1134/S0015462819040037

24. Johnston, C. O., Mazaheri, A., Gnoffo, P., Kleb, B., Sutton, K., Prabhu, D., Brandis, A. M., Bose, D., "Radiative heating uncertainty for hyperbolic Earth entry, part 1: flight simulation modeling and uncertainty," J. Spacecraft \& Rockets, Vol. 50, No. 1, 2013, pp. 19-38. https://doi.org/10.2514/1.A32254

25. Brykina, I. G., Bragin, M. D., "On models of meteoroid disruption into the cloud of fragments," Planetary \& Space Sci., Vol. 187, No. 104942, 2020. https://doi.org/10.1016/j.pss.2020.104942

26. Turchak, L. I., Osnovy chislennykh metodov: uchebnoye posobiye dlya studentov vuzov, M.: Vyssh. shk., $1987,320 \mathrm{p}$.

27. Brykina, I. G., Tirskiy, G. A., "Mass loss and light curve of a large meteoroid. Analytical solution," J. Applied Mathematics and Mechanics, Vol. 81, 2017, pp. 395-408. https://doi.org/10.1016/j.jappmathmech.2018.03.008.

28. Suttles, J. T., Sullivan, E. M., Margolis, S. B., "Curve fits of predicted inviscid stagnation-point radiative heating rates, cooling factors, and shock standoff distances for hyperbolic earth entry," NASA TN D-7622, 1974. https://ntrs.nasa.gov/archive/nasa/casi.ntrs.nasa.gov/19740021216.pdf.

Статья поступила в редакцию 17 октября 2020 г. 